\title{
TAUBERIAN THEOREMS FOR A POSITIVE DEFINITE FORM, WITH APPLICATIONS TO A VOLTERRA EQUATION
}

\author{
BY
}

\section{OLOF J. STAFFANS}

ABSTRACT. We study the relation between the condition

$$
\sup _{T>0} \int_{[0, T]} \bar{\varphi}(t) \int_{[t-T, t]} \varphi(t-s) d \nu(s) d t<\infty
$$

and the asymptotic behavior of the bounded function $\varphi$ when $\nu$ is a positive definite measure. Earlier we have proved that if $\nu$ is strictly positive definite and $\varphi$ satisfies a tauberian condition, then $\varphi(t) \rightarrow 0$ as $t \rightarrow \infty$. Here we characterize the spectrum of the limit set of $\varphi$ in the case when $\nu$ is not strictly positive definite. Applying this theory to a nonlinear Volterra equation we get some new results on the asymptotic behavior of its bounded solutions.

Introduction. This paper is devoted to the following problem: Let $\nu$ be a positive definite measure on $(-\infty, \infty)$ (i.e. a positive definite distribution of order zero, e.g. a positive definite function in the sense of Bochner), let $\varphi$ be a bounded function on $(-\infty, \infty)$, and suppose that.

$$
\sup _{T>0} \int_{[0, T]} \bar{\varphi}(t) \int_{[t-T, t]} \varphi(t-s) d v(s) d t<\infty .
$$

What can then be said about the behavior of $\varphi$ at infinity? Our interest in this question arises from the fact that its answer can be used in the study of the asymptotic behavior of the bounded solutions to a nonlinear Volterra integrodifferential equation (Lemma 7.1 below). This problem has been investigated in [22], and it also appears in different versions as a subproblem e.g. in [2], [11], [12] , [14]-[16] and [21] (see e.g. [11, line (2.3)] and [12, lines (2.6) and (2.11)]), which basically are devoted to the study of nonlinear Volterra equations ([14] and [15] are phrased in Hilbert space setting). However, as they apply to this particular question, all these papers (except for [12] and [14], cf. the more detailed discussion below) only give sufficient conditions on the measure $v$ which imply that $\varphi(t) \rightarrow 0$ in some sense (not necessarily pointwise convergence) as $t \rightarrow \infty$.

Received by the editors April 17, 1975.

AMS (MOS) subject classifications (1970). Primary 42-00, 42A68, 42A88, 45D05, 45G99, 45J05, 45M05.

Key words and phrases. Tauberian theorem, positive definite, quadratic form, asymptotic behavior, limit set, asymptotic spectrum, removable zeros, nonlinear Volterra equation. 
Inspired by the work of Levin and Shea [10], in which the asymptotic behavior of the solutions of some nonlinear integral equations is described in terms of the solutions of a limit equation, we approach the problem in a new way. We study the limit set $\Gamma(\varphi)$ of the given function $\varphi$, defined as follows (provided $\varphi$ satisfies a tauberian condition):

$$
\begin{aligned}
& \Gamma(\varphi)=\left\{\psi \in L^{\infty}(-\infty, \infty) \mid \text { there exists a sequence } t_{k} \rightarrow \infty\right. \text { such that } \\
& \left.\qquad \tau_{t_{k}} \varphi \rightarrow \psi \text { uniformly on compact subsets of }(-\infty, \infty)\right\}
\end{aligned}
$$

(here we use the notation $\left.\tau_{h} \varphi(t)=\varphi(t+h)(-\infty<t, h<\infty)\right)$. In terms of the limit set $\Gamma(\varphi)$, all the references listed above on how (1) relates to the behavior of $\varphi$ at infinity only give sufficient conditions on $\nu$ which imply that $\Gamma(\varphi)=\{0\}$, or that $\Gamma(\varphi)$ only contains constant functions ([12] and [14]). These sufficient conditions have been strong enough to imply that the (distribution) Fourier transform $\hat{\nu}$ of $\nu$ is (nonnegative and) locally bounded away from zero (with the exception of [12] and [14], where $\hat{\nu}(0)=0$ is possible; in [12] also some other zeros can occur in $\hat{\nu}$, but then additional properties of $\varphi$ (the fact that $\varphi$ arises from a Volterra equation) are used to get the desired result). It turns out that in the general case, i.e. when $\hat{v}$ is not locally bounded away from zero, the behavior of $\varphi$ at infinity can be related to the zeros of $\hat{\nu}$ (see $\S 1$ for precise definitions): The spectrum of the set $\Gamma(\varphi)$ is contained in the zero set $Z(\nu)$ of $\hat{\nu}$ (Theorem 3.1 below).

In addition to the result of Theorem 3.1, which is not quite sharp unless $\hat{\nu}$ is smooth enough, we have been able to characterize completely the relation between (1) and the size of $\hat{v}$ in a neighborhood of an isolated point of $Z(v)$ (Theorems 4.1-4.2 below; see also Remark 4.1). The basic interpretation of Theorems 4.1-4.2 is the following: An isolated point $\omega_{0}$ of $Z(\nu)$ can belong to the spectrum of $\Gamma(\varphi)$ for some $\varphi$ satisfying (1) if and only if $\hat{\nu}$ is "small" in a neighborhood of $\omega_{0}$. The exact meaning of "big" (i.e. not "small") is given by (4.1) below, or equivalently by (6.1)-(6.2) below; but if $\hat{\nu}$ is given by a continuous function $u$, then "small" essentially stands for a second, or higher, order zero of $u$, whereas zeros where the derivative of $u$ is discontinuous are "big" (the function $u$ always has a minimum at $\omega_{0}$ by the requirement that $v$ be positive definite). In particular, if $u$ is smooth enough, then all zeros are of the "small" type (Propositions 6.1 and 6.2). This fact can be used to prove a partial converse to [22, Theorem 2.1] (see Corollary 6.1 below).

Applying our results to a nonlinear Volterra integrodifferential equation we get a description of the asymptotic behavior of its bounded solutions in terms of the spectrum of a limit set (Proposition 7.1 below), which has been done previously only in very special cases. Some theorems of Levin and Shea [10] do 
use a description quite similar to ours, but they only apply to a linear integral equation, and they require stronger boundedness conditions on the kernel (however, they can then instead use a sharper Fourier transform condition, and also allow bigger perturbation functions). Another major difference between our work and that of Levin and Shea is that in place of the limit equation we use the quadratic form (1) to obtain knowledge about the behavior of the solutions at infinity. If one also uses the limit equation, then Proposition 7.1 can be substantially refined (provided one has some growth conditions on the measure $\nu$ ), and we intend to return to this question elsewhere.

A result comparable to $[5$, Corollary 2.2$],[12$, Theorem 1$]$ and $[14$, Theorem 4.1] (restricted to the real line), which consider a nondifferentiated Volterra equation, can be obtained from Proposition 7.1, but we defer a demonstration of this fact to a later date. We shall also discuss elsewhere how Proposition 7.1 relates to some results on a particular equation with a piecewise linear kernel, first studied by Levin and Nohel (see e.g. [8, Theorem 1] and [3, Theorem 1(ii)(iii)]).

A recent study by Londen [13], which gives lower bounds on the total variation of oscillatory solutions of a Volterra equation, as well as some sufficient conditions for the solutions to approach a constant value, overlaps Proposition 7.1.

In §7 we also give an explicit example of a Volterra equation, which illustrates the force of Proposition 7.1. Here we have a zero in the Fourier transform of the kernel, but this does not affect the behavior of the solution of (7.2) below, as long as the perturbation term $f$ in (7.2) is integrable. We show that the integrability condition on $f$ is very sharp, i.e. it cannot be replaced by $f(t)=$ $O\left(t^{-1}\right)(t \rightarrow \infty)$. This result is new even in the case when the equation is linear.

1. Definitions and notations. We shall basically use the same notations as in [22], but for the reader's convenience we review them below.

The following special symbols are used:

$\chi_{I}$ : the characteristic function of the interval $I$,

$\sigma(\varphi), \sigma(\Phi)$ : see Definitions 1.6-1.7,

$S(\varphi), \bar{S}(\varphi), \Gamma(\varphi)$ : see Definition 1.8 ,

$Z(\nu)$ : see Definition 1.5.

In addition to the ordinary $L^{p}$ spaces we shall need the following function, measure or distribution spaces (for simplicity we omit the interval of definition):

$M, P, P D, S P D$ : see Definitions 1.1, 1.2 and 1.4,

$C$ : continuous functions,

$C_{u}$ : uniformly continuous functions,

$S:$ rapidly decreasing testfunctions,

$S^{\prime}$ : tempered distributions. 
The next list explains the meaning of certain operators (here $\psi$ belongs to some function space for which the definitions make sense, and $u$ to the corresponding dual space):

$\langle\psi, u\rangle: u$ evaluated at $\psi$,

$\bar{\psi}$ : complex conjugate of $\psi$.

$\bar{A}$ : closure of $A$,

$\hat{\psi}: \hat{\psi}(\omega)=\int_{-\infty}^{\infty} e^{-i \omega t} \psi(t) d t(-\infty<\omega<\infty)$,

$\hat{u}:\langle\psi, \hat{u}\rangle=\langle\hat{\psi}, u\rangle$,

$\tilde{\psi}: \widetilde{\psi}(t)=\bar{\psi}(-t)(-\infty<t<\infty)$,

$\tilde{u}:\langle\psi, \tilde{u}\rangle=\langle\widetilde{\psi}, u\rangle$,

$\tau_{h} \psi: \tau_{h} \psi(t)=\psi(t+h)(-\infty<t, h<\infty)$,

$f * g:(f * g)(t)=\int_{-\infty}^{\infty} f(t-s) g(s) d s\left(f \in L^{p}(-\infty, \infty), g \in L^{q}(-\infty, \infty)\right.$, where $p$ and $q$ are conjugate exponents),

$|\nu|$ : the total variation measure of $\nu$.

We identify a measure $\mu$ (or a function) with a distribution by the formula

$$
\langle\psi, \mu\rangle=\int_{-\infty}^{\infty} \psi(t) d \mu(t) \quad(\psi \in S \text { with compact support }) .
$$

We extend all functions and measures, defined on an interval $I$, to $(-\infty, \infty)$ by zero outside $I$, unless we give a specific statement to the contrary.

The following set of definitions is a slightly modified version of the corresponding definitions in [22]:

DEFinition 1.1. A locally finite (Radon) measure $v$ on $(-\infty, \infty)(\nu \in$ $M(-\infty, \infty))$ is a complex-valued set function which restricted to subsets of compact sets is a (finite) complex Borel measure. The subclass of locally finite, positive measures is denoted by $P(-\infty, \infty)$.

Definition 1.2. A measure $\nu \in M(-\infty, \infty)$ is positive definite $(\nu \in$ $P D(-\infty, \infty))$ if

$$
\int_{-\infty}^{\infty} \bar{\varphi}(t) \int_{-\infty}^{\infty} \varphi(t-s) d \nu(s) d t \geqslant 0
$$

for every $\varphi \in L^{2}(-\infty, \infty)$ with compact support.

We recall the following theorem (see [22, Theorem 1.2] and the line following it):

Theorem 1.1 (Schwartz). A measure $\nu \in M(-\infty, \infty)$ is positive definite if and only if $\nu \in S^{\prime}$ and $\hat{\nu} \in P(-\infty, \infty)$.

We shall frequently use this theorem without a specific reference to it.

Definition 1.3. A measure $\lambda \in M(-\infty, \infty)$ is strictly positive at a point $\omega$ if there exists $\epsilon>0$ such that the measure $\lambda_{\epsilon}$ defined by $d \lambda_{\epsilon}(t)=d \lambda(t)-$ $\epsilon d t$ is positive in $(\omega-\epsilon, \omega+\epsilon)$. 
Definition 1.4. A measure $\nu \in P D(-\infty, \infty)$ is strictly positive definite $(\nu \in \operatorname{SPD}(-\infty, \infty))$ if $\hat{\nu}$ is strictly positive everywhere (cf. [22, Theorem 2.2]).

Definition 1.5. The spectral set $Z(\nu)$ of a measure $\nu \in P D(-\infty, \infty)$ is the complement of the set where $\hat{\nu}$ is strictly positive.

The next definition concerns the spectrum of a bounded function:

Definition 1.6. The spectrum $\sigma(\varphi)$ of a bounded function $\varphi$ is the support of the (distribution) Fourier transform $\hat{\varphi}$.

This definition is equivalent to several other definitions commonly used. In particular, one has

$$
\sigma(\varphi)=\bigcap_{f \in A}\{\omega \in(-\infty, \infty) \mid \hat{f}(\omega)=0\}
$$

where $A=\left\{f \in L^{1}(-\infty, \infty) \mid f * \varphi \equiv 0\right\}$. That $\sigma(\varphi)$ is contained in the right-hand side of (1.1) follows from [19, Theorem 9.3], and the other inclusion is trivial (if $\omega \notin \sigma(\varphi)$, then there exists $\epsilon>0$ such that $(\omega-\epsilon, \omega+\epsilon) \cap \sigma(\varphi)=\varnothing$; choose $f \in S$ so that $\sigma(f) \in(\omega-\epsilon, \omega+\epsilon), \hat{f}(\omega) \neq 0$; then use $[19$, Theorem $7.19(c)])$. For a discussion on different notions of spectra we refer the reader to [10, pp. 298-299].

DEFINITION 1.7. The spectrum of a set $\Phi \subset L^{\infty}(-\infty, \infty)$ is defined as $\sigma(\Phi)$ $=\overline{U_{\varphi \in \Phi} \sigma(\varphi)}$.

Two basic concepts in this study are the set of left-translates $S(\varphi)$ and the limit set $\Gamma(\varphi)$ of a bounded function:

Definition 1.8. Let $\varphi \in L^{\infty}(-\infty, \infty)$. The set of left-translates $S(\varphi)$ is given by

$$
S(\varphi)=\left\{\tau_{h} \varphi \mid h \geqslant 0\right\}
$$

The notation $\bar{S}(\varphi)$ stands for the weak ${ }^{*}$ closure in $L^{\infty}(-\infty, \infty)$ of $S(\varphi)$. The limit set $\Gamma(\varphi)$ of $\varphi$ is given by

$$
\begin{aligned}
& \Gamma(\varphi)=\left\{\psi \in L^{\infty}(-\infty, \infty) \mid \text { there exists a sequence } t_{k} \rightarrow \infty\right. \\
& \text { such that } \left.\tau_{t_{k}} \varphi \rightarrow \psi \text { weak* in } L^{\infty}(-\infty, \infty)\right\} .
\end{aligned}
$$

REMARK 1.1. We shall study the set $\sigma(\Gamma(\varphi))$ extensively for functions $\varphi$ satisfying (1). It may be worth while mentioning that the inclusion $\sigma(\Gamma(\varphi)) \subset$ $\sigma(\varphi)$ always holds. This follows from the fact that every $\psi \in \Gamma(\varphi)$ is the limit in $S^{\prime}$ of a sequence of translates of $\varphi$, and the supports of the Fourier transforms of these translates are all equal to $\sigma(\varphi)$.

2. The topology of $\bar{S}(\varphi)$ and $\Gamma(\varphi)$. Since $\bar{S}(\varphi)$ is defined as the weak* closure in $L^{\infty}(-\infty, \infty)$ of the set of left-translates $S(\varphi)$ of $\varphi$, the natural topology on $\bar{S}(\varphi)$ is the induced weak* topology. The following lemma details some of 
the properties of $\bar{S}(\varphi)$ under this topology:

LEMMA 2.1. Let $\varphi \in L^{\infty}(-\infty, \infty)$. Give $\bar{S}(\varphi)$ the induced weak topology. Then $\bar{S}(\varphi)$ is compact, connected and metrizable. The limit set $\Gamma(\varphi)$ is a nonempty, closed and connected subset of $\bar{S}(\varphi)$ (hence compact), and the distance from $\tau_{t} \varphi$ to $\Gamma(\varphi)$ tends to zero as $t \rightarrow \infty$.

Proof of Lemma 2.1. That $\bar{S}(\varphi)$ is compact and metrizable follows from [19, Theorems 3.16 and $4.3(\mathrm{c})]$. Since translation is a weak* continuous operation in $L^{\infty}(-\infty, \infty)$ (this follows from the fact that translation is continuous in $L^{1}(-\infty, \infty)$ ), the set $S(\varphi)$ is a weak* continuous image of the connected set [0, $\infty)$ and hence connected [17, Proposition 8, p. 153]. But $S(\varphi)$ is dense in $\bar{S}(\varphi)$, and thus also $\bar{S}(\varphi)$ is connected [17, Problem 31, p. 153].

To prove that the limit set $\Gamma(\varphi)$ has the properties listed in Lemma 2.1 one can now simply repeat the familiar argument for the corresponding properties of the limit set of a bounded, continuous curve in the plane (here we instead have the weak* continuous curve $\tau_{t} \varphi(t \geqslant 0)$ in a weak* compact subset of $L^{\infty}(-\infty, \infty)$, and the topology is metrizable). We therefore leave the completion of the proof to the reader.

In $[6$, line (2.3)] a concept similar to the limit set $\Gamma(\varphi)$ is defined. However, there only certain uniformly continuous functions $\varphi$ are considered. The corollary to the following lemma simply says that in the case of a uniformly continuous function $\varphi$ these two concepts agree.

Lemma 2.2. Let $\varphi \in L^{\infty}(-\infty, \infty) \cap C_{u}(-\infty, \infty)$. Then $\bar{S}(\varphi) \subset L^{\infty}(-\infty, \infty) \cap$ $C_{u}(-\infty, \infty)$, and the weak ${ }^{*}$ topology on $\bar{S}(\varphi)$ (regarded as a subset of $L^{\infty}(-\infty, \infty)$ ) is equivalent to the topology of uniform convergence on compact subsets of $(-\infty$, $\infty)$ (regarding $\bar{S}(\varphi)$ as a subset of $C(-\infty, \infty)$ ).

The topology of uniform convergence on compact subsets is described e.g. in [19, Example 1.44].

CoRollary 2.1. If $\varphi \in L^{\infty}(-\infty, \infty) \cap C_{u}[0, \infty)$, then $\Gamma(\varphi)=\left\{\psi \in L^{\infty}(-\infty, \infty) \cap C_{u}(-\infty, \infty) \mid\right.$ there exists a sequence $t_{k} \rightarrow \infty$ such that $\tau_{t_{k}} \varphi \rightarrow \psi$ uniformly on compact subsets of $\left.(-\infty, \infty)\right\}$.

Note that $\Gamma(\varphi)$ is independent of the values of $\varphi$ on the negative half line, so in order to prove Corollary 2.1 it suffices to redefine $\varphi$ on $(-\infty, 0)($ e.g. $\varphi(t)=\varphi(0), t<0)$, so that $\varphi$ is uniformly continuous on $(-\infty, \infty)$, and then apply Lemma 2.2 .

REMARK 2.1. In Corollary 2.1 the uniform continuity of $\varphi$ on $[0, \infty)$ only plays the role of a tauberian condition at $+\infty$, and it could be replaced by other tauberian conditions, e.g. the one used in [10, Lemma 3.2, p. 75].

Proof of Lemma 2.2. Let $T(\varphi)$ be the closure in $C(-\infty, \infty)$ of $S(\varphi)$ in the top- 
ology of uniform convergence on compact sets. We first want to show that $\bar{S}(\varphi)=$ $T(\varphi)$. Take $\psi \in T(\varphi)$. The topology of uniform convergence on compact sets is metrizable [19, Example 1.44], so there is a sequence $\psi_{k} \in S(\varphi)$ converging to $\psi$ uniformly on compact subsets of $(-\infty, \infty)$. Lebesgue's dominated convergence theorem then shows that $\psi_{k} \rightarrow \psi$ weak ${ }^{*}$ in $L^{\infty}(-\infty, \infty)$, and hence $\psi \in \bar{S}(\varphi)$.

Conversely, let $\psi \in \bar{S}(\varphi)$. The weak ${ }^{*}$ topology on $\bar{S}(\varphi)$ being metrizable there exists a sequence $\psi_{k} \in S(\varphi)$ converging to $\psi$ weak ${ }^{*}$ in $L^{\infty}(-\infty, \infty)$. However, the sequence $\psi_{k}$ is uniformly bounded and equicontinuous, and hence by Ascoli's theorem every subsequence of $\psi_{k}$ contains a subsequence converging uniformly on compact subsets of $(-\infty, \infty)$ to some continuous function. But uniform convergence on compact sets together with uniform boundedness implies weak* convergence in $L^{\infty}(-\infty, \infty)$ (use Lebesgue's dominated convergence theorem), and so the limit function must be $\psi$ (modulo a set of measure zero). This is true for every subsequence, and so the complete sequence converges to $\psi$ uniformly on compact sets. That $\psi$ is uniformly continuous is a consequence of the uniform continuity of $\varphi$, and the uniform convergence on compact sets; in fact $\psi$ has a modulus of continuity which is bounded above by the modulus of continuity of $\varphi$. In particular, the set $\bar{S}(\varphi)$ is equicontinuous.

We have now proved that $\bar{S}(\varphi)=T(\varphi)$. Moreover, essentially the same argument as the one above shows that the identity mapping on $\bar{S}(\varphi)$ between the two topologies is sequentially continuous both ways (for that one has to take sequences $\psi_{k} \in \bar{S}(\varphi)$ instead of $S(\varphi)$, but the rest of the argument remains the same). Since both topologies are metrizable on $\bar{S}(\varphi)$ this means that the identity mapping is continuous both ways, and thus the two topologies are equivalent.

REMARK 2.2. One could use $[10$, Lemma 3.1, p. 72$]$ together with Lemmas 2.1-2.2 above to show that a function $\varphi \in L^{\infty}(-\infty, \infty) \cap C_{u}(-\infty, \infty)$ can be written in the form

$$
\varphi(t)=\sum_{k=1}^{\infty} \psi_{k}(t) y_{k}(t)+\eta(t) \quad(t \geqslant 0)
$$

where $\eta(t) \rightarrow 0(t \rightarrow \infty), y_{k} \in \Gamma(\varphi)$, and $\left\{\psi_{k}\right\}$ is a " $\psi$-sequence" as defined in [10], i.e. basically a smooth partition of unity that varies slower and slower as $t \rightarrow \infty$ (see also $[10$, p. 572]). We shall not need this representation below (except in the trivial case when $\Gamma(\varphi)=\{0\}$ ), and we therefore omit the precise statement.

3. On the spectrum of $\Gamma(\varphi)$. We proved in [22] that strict positive definiteness of a measure $\nu$ together with boundedness of $\varphi$ on $(-\infty, \infty)$ and the condition (1) forces the left-translates $\tau_{t} \varphi$ of $\varphi$ to converge to zero weak ${ }^{*}$ in $L^{\infty}(-\infty, \infty)$ as $t \rightarrow \infty$ (to simplify the notations slightly we here use measures $\nu \in P D(-\infty, \infty)$ in. stead of measures $\mu \in P D[0, \infty)$; cf. [22, Theorem 1.1]). In terms of the set $\Gamma(\varphi)$ this means that $\Gamma(\varphi)=\{0\}$, i.e. the zero function in $L^{\infty}(-\infty, \infty)$. We shall 
now generalize this result by proving a theorem on the spectrum of $\Gamma(\varphi)$ in the case when $\nu$ is positive definite, but not necessarily strictly positive definite, and (1) holds.

THEOREM 3.1. Let $\nu \in P D(-\infty, \infty), \varphi \in L^{\infty}(-\infty, \infty)$, and suppose that (1) holds. Then $\sigma(\Gamma(\varphi)) \subset Z(\nu)$.

This theorem, combined with Lemmas 2.1-2.2 above, contains the result of [22, Theorem 2.1] as a special case. If $\nu$ is strictly positive definite, then $Z(\nu)=\varnothing$, and so $\Gamma(\varphi)=\{0\}$ (combine [19, Theorem 6.24(b)] with the Fourier inversion theorem). The conclusion of [22, Theorem 2.1] now follows if one applies Lemmas 2.1-2.2 (use also [22, Lemma 1.1 and Theorems 1.1, 2.2]).

Proof of Theorem 3.1. By [22, Lemma 2.2a] combined with Theorem 1.1 there exists a function $a \in S$ such that $\hat{a}(\omega)=0(\omega \in Z(\nu)), \hat{a}(\omega)>0(\omega$ $\notin Z(\nu))$, and the measure $\lambda$ defined by $d \lambda(t)=d \nu(t)-a(t) d t(-\infty<t<\infty)$ is positive definite (i.e. $\lambda \in P D(-\infty, \infty)$ ). This together with (1) gives

$$
\sup _{T>0} \int_{0}^{T} \bar{\varphi}(t) \int_{t-T}^{t} \varphi(t-s) a(s) d s d t<\infty .
$$

We can now use a trivial modification of the proof of [21, Lemma 2] (i.e. add complex conjugates in the appropriate places) to get

$$
\lim _{t \rightarrow \infty} \int_{0}^{\infty} a(t-s) \varphi(s) d s=0 .
$$

This together with $a \in L^{1}(-\infty, \infty), \varphi \in L^{\infty}(-\infty, \infty)$ then gives

$$
\lim _{t \rightarrow \infty} \int_{-\infty}^{\infty} a(t-s) \varphi(s) d s=0 .
$$

Now take any $\psi \in \Gamma(\varphi)$, and let $s_{k} \rightarrow \infty(k \rightarrow \infty)$ be a sequence such that $\tau_{s_{k}} \varphi \rightarrow \psi$ weak $^{*}$ in $L^{\infty}(-\infty, \infty)(k \rightarrow \infty)$. Fix $t \in(-\infty, \infty)$. Then

$$
0=\lim _{k \rightarrow \infty} \int_{-\infty}^{\infty} a\left(t+s_{k}-s\right) \varphi(s) d s=\lim _{k \rightarrow \infty} \int_{-\infty}^{\infty} a(t-s)\left(\tau_{s_{k}} \varphi\right)(s) d s .
$$

But $a \in L^{1}(-\infty, \infty)$, and $\tau_{s_{k}} \varphi \rightarrow \psi$ weak ${ }^{*}$ in $L^{\infty}(-\infty, \infty)$, and so

$$
0=\lim _{k \rightarrow \infty} \int_{-\infty}^{\infty} a(t-s)\left(\tau_{s_{k}} \varphi\right)(s) d s=\int_{-\infty}^{\infty} a(t-s) \psi(s) d s \quad(-\infty<t<\infty) .
$$

The fact that $\hat{a}(\omega) \neq 0(\omega \notin Z(\nu))$ together with (1.1) then gives $\sigma(\psi) \subset Z(\nu)$. This is true for every $\psi \in \Gamma(\varphi)$, and since $Z(\nu)$ is closed we find that $\sigma(\Gamma(\varphi)) \subset$ $Z(\nu)$. This completes the proof of Theorem 3.1.

COROLlARY 3.1. If in addition to the assumption of Theorem 3.1 we have $Z(\nu)$ countable, and either $Z(\nu)$ compact or $\varphi \in C_{u}(-\infty, \infty)$, then the functions in $\Gamma(\varphi)$ are uniformly almost periodic with Fourier exponents in $Z(v)$.

This is a consequence of $[4$, Theorem 5.22, p. 168] and [10, Proposition 8.1(ii), p. 297] (use also Lemma 2.2 above).

COROLlARY 3.2. If in addition to the assumption of Theorem 3.1 the set 
$Z(\nu)$ is finite, then the functions in $\Gamma(\varphi)$ are trigonometric polynomials.

This follows from Theorem 3.1 e.g. if one applies Lemma 5.2 below a finite number of times.

COROLlARY 3.3. If in addition to the assumption of Theorem 3.1 there exists $\omega_{0}>0$ such that $Z(\nu) \subset\left\{n \omega_{0} \mid n=0, \pm 1, \pm 2, \ldots\right\}$, then the functions in $\Gamma(\varphi)$ are periodic with period $2 \pi / \omega_{0}$.

This is true because the Fourier transform $\hat{\psi}$ of any $\psi \in \Gamma(\varphi)$ will consist of point masses at the points $\left\{n \omega_{0}\right\}(n=0, \pm 1, \pm 2, \ldots)$ (this follows from Lemma 5.2 below), and therefore $\hat{\psi}$ remains invariant under multiplication by the function $e^{i 2 \pi \omega / \omega_{0}}(-\infty<\omega<\infty)$, which means that $\tau_{2 \pi / \omega_{0}} \psi=\psi$, i.e. $\psi$ is periodic.

4. Further restrictions on $\sigma(\Gamma(\varphi))$. The inclusion $\sigma(\Gamma(\varphi)) \subset Z(\nu)$ in Theorem 3.1 can be proper, and it is easy to construct examples with particular measures $\nu$ and functions $\varphi$ where this is the case (take e.g. $\nu=0, \varphi(t)=e^{i \omega t}$ ). A more meaningful question is whether it is possible to find points in $Z(v)$ which do not belong to $\sigma(\Gamma(\varphi))$ for any $\varphi$ satisfying (1), i.e. whether it is possible to replace $Z(\nu)$ in Theorem 3.1 by a (proper) subset of $Z(\nu)$. The answer is negative, if $\hat{\nu}$ is smooth enough, but for a less smooth $\hat{\nu}$ it is in general affirmative. The following theorem, which is independent of Theorem 3.1, provides the key to the affirmative answer, whereas its converse, Theorem 4.2 below, together with Propositions 6.1-6.2 below also serves as a converse to Theorem 3.1 for smooth $\hat{v}$.

TheOREM 4.1. Let $\nu \in P D(-\infty, \infty), \varphi \in L^{\infty}(-\infty, \infty)$, and let (1) hold. Moreover, suppose that for some $\omega_{0} \in(-\infty, \infty)$,

$$
\sup _{T>0} \int_{-\infty}^{\infty} K_{T}\left(\omega-\omega_{0}\right) d \hat{v}(\omega)=\infty
$$

where

$$
K_{T}(s)=\left\{\begin{array}{l}
1 / 2 T^{2}, \quad s=0, \\
s^{-2}(1-\cos (T s)), \quad s \neq 0 .
\end{array}\right.
$$

Then $\omega_{0}$ is not an isolated point of $\sigma(\Gamma(\varphi))$.

The proof of Theorem 4.1 is rather long, and we have therefore deferred it to $\S 5$.

REMARK 4.1. Although the integration in (4.1) is extended over $(-\infty, \infty)$, this is still a purely local condition on $\hat{v}$ at the point $\omega_{0}$ for the following reason: Pick any $\epsilon>0$. Then 


$$
\begin{aligned}
& \left(\int_{\left(-\infty, \omega_{0}-\epsilon\right)}+\int_{\left(\omega_{0}+\epsilon, \infty\right)}\right)\left(\omega-\omega_{0}\right)^{-2} d \hat{\nu}(\omega) \\
& \quad=\sum_{n=1}^{\infty}\left(\int_{\left[\omega_{0}-(n+1) \epsilon, \omega_{0}-n \epsilon\right)}+\int_{\left(\omega_{0}+n \epsilon, \omega_{0}+(n+1) \epsilon\right]}\right)\left(\omega-\omega_{0}\right)^{-2} d \hat{\nu}(\omega) \\
& \leqslant \epsilon^{-2} \sum_{n=1}^{\infty} n^{-2}\left\{\hat{\nu}\left(\left[\omega_{0}-(n+1) \epsilon, \omega_{0}-n \epsilon\right)\right)+\hat{v}\left(\left(\omega_{0}+n \epsilon, \omega_{0}+(n+1) \epsilon\right]\right)\right\},
\end{aligned}
$$

and by [22, Proposition 1.1] this sum is always finite.

It is easy to see that (4.1) is weaker than strict positivity of $\hat{v}$ at $\omega_{0}$, e.g. (4.1) holds whenever the derivative of $\hat{v}$ with respect to Lebesgue measure at the point $\omega_{0}$ is nonzero (use the fact that $K_{T}\left(\omega-\omega_{0}\right) \geqslant 2 \pi^{-2} T^{2}\left(\left|\omega-\omega_{0}\right| \leqslant\right.$ $\pi T^{-1}$ ), and let $T \rightarrow \infty$ ). However, (4.1) does not exclude a zero derivative of $\hat{\nu}$ at $\omega_{0}$, as long as the derivative of $\hat{\nu}$ does not "come in tangentially" at $\omega_{0}$. In $\S 6$ we shall further discuss what (4.1) means in terms of smooth functions $\hat{\nu}$.

Maybe the most remarkable feature of the condition (4.1) is that it is also necessary, as the following converse to Theorem 4.1 shows:

THEOREM 4.2. Let $\nu \in P D(-\infty, \infty)$, and suppose that for some $\omega_{0} \in$ $(-\infty, \infty)$,

$$
\sup _{T>0} \int_{-\infty}^{\infty} K_{T}\left(\omega_{0}-\omega\right) d \hat{\nu}(\omega)<\infty,
$$

with $K_{T}$ defined by (4.2). Then there exists $\varphi \in L^{\infty}(-\infty, \infty) \cap C_{u}(-\infty, \infty)$ (e.g. $\left.\varphi(t)=e^{i \omega_{0} t}\right)$ such that $(1)$ holds, and $\sigma(\Gamma(\varphi))=\left\{\omega_{0}\right\}$.

The proof of this theorem uses the following lemma, which will be proved in $\S 5$ :

LEMMA 4.1. Let $\varphi \in L^{2}(-\infty, \infty)$ have compact support, and suppose that $\nu \in P D(-\infty, \infty)$. Then

$$
\overline{\langle\varphi * \widetilde{\varphi}}, \nu\rangle=\frac{1}{2 \pi}\left\langle|\hat{\varphi}|^{2}, \hat{v}\right\rangle .
$$

A weaker version of this lemma is contained in the proof of $[22$, Lemma 6.1].

Proof of Theorem 4.2. Define $\varphi(t)=e^{i \omega_{0} t}(-\infty<t<\infty)$. By Remark 1.1 we have $\sigma(\Gamma(\varphi)) \subset \sigma(\varphi)=\left\{\omega_{0}\right\}$, and since it is trivial to show that in this case $\varphi \in \Gamma(\varphi)$, we get $\sigma(\Gamma(\varphi))=\left\{\omega_{0}\right\}$. It only remains to show that (1) holds. However, by Lemma 4.1 and the fact that

$$
\left|\int_{0}^{T} e^{-i\left(\omega-\omega_{0}\right) t} d t\right|^{2}=2 K_{T}\left(\omega-\omega_{0}\right),
$$

the conditions (1) and (4.3) are equivalent. This completes the proof of Theorem 4.2 . 
REMARK 4.2 We want to emphasize the connection between (1) and (4.1) that comes up in the proof of Theorem 4.2 (fix some $\nu \in P D(-\infty, \infty)$ ): The condition (4.1) is true if and only if the condition (1) is false for the function $\varphi(t)=$ $e^{i \omega_{0} t}(-\infty<t<\infty)$. Thus Theorem 4.1 can also be phrased as follows: If the quadratic integral in (1) is unbounded as $T \rightarrow \infty$ for $\varphi(t)=e^{i \omega_{0} t}(-\infty<t<$ $\infty)$, then it is also unbounded as $T \rightarrow \infty$ for any $\varphi \in L^{\infty}(-\infty, \infty)$ such that $\omega_{0}$ is an isolated point of $\sigma(\Gamma(\varphi))$.

Combining Theorems 3.1 and 4.1 one gets

ThEOREM 4.3. Let $\nu \in P D(-\infty, \infty), \varphi \in L^{\infty}(-\infty, \infty)$, and let (1) hold. Moreover, let $O \subset(-\infty, \infty)$ be an open set such that $O \cap Z(\nu)$ is countable, and such that (4.1) holds for every $\omega_{0} \in O \cap Z(\nu)$. Then $0 \cap \sigma(\Gamma(\varphi))=\varnothing$. In particular, if $0=(-\infty, \infty)$, then $\Gamma(\varphi)=\{0\}$.

Note that the last statement trivially follows from the previous statement, since no nonzero distribution has an empty support.

Proof of TheOREM 4.3. It clearly suffices to show that $I \cap \sigma(\Gamma(\varphi))=\varnothing$ for every closed interval $I \subset 0$. Take any such interval $I$. Slightly enlarging $I$ if necessary we can suppose that the endpoints of $I$ do not belong to $Z(v)$. By Theorems 3.1 and 4.2, the set $I \cap \sigma(\Gamma(\varphi))$ is closed, countable, and it has no isolated points. Hence it is a perfect set. But every nonempty perfect set has more than countably many points, and thus $I \cap \sigma(\Gamma(\varphi))=\varnothing$. This completes the proof of Theorem 4.3.

Combining Theorems 4.2 and 4.3 with Lemma 2.1 one obviously gets

Corollary 4.1. Let $\nu \in P D(-\infty, \infty)$, and suppose that $Z(\nu)$ is countable. Then every $\varphi \in L^{\infty}(-\infty, \infty)$ for which (1) holds satisfies $\tau_{t} \varphi \rightarrow 0$ weak $k^{*}$ in $L^{\infty}(-\infty, \infty)$ as $t \rightarrow \infty$ if and only if $(4.1)$ is true for every $\omega_{0} \in(-\infty, \infty)$.

A different version, where the functions $\varphi$ are uniformly continuous, and weak* convergence in $L^{\infty}(-\infty, \infty)$ is replaced by pointwise convergence, could also be given (cf. Corollary 2.1).

5. Proofs of Lemma 4.1 and Theorem 4.1. We begin with the proof of Lemma 4.1, since it will be needed in the proof of Theorem 4.1.

Proof of Lemma 4.1. Choose $T$ so large that the support of $\varphi$ lies in $[-T+1, T-1]$. Pick any $\eta \in S$ with support in $(-1,1)$ and $\hat{\eta}(0)=1$. Define $\eta_{n}(t)=n \eta(n t)(-\infty<t<\infty), \varphi_{n}=\eta_{n} * \varphi(n=1,2, \ldots)$. Then $\varphi_{n} \in S$ vanishes outside $(-T, T)(n \geqslant 1)$, and $\varphi_{n} \rightarrow \varphi$ is $L^{2}(-\infty, \infty)$ as $n \rightarrow \infty$. Thus $\varphi_{n} * \widetilde{\varphi}_{n}$ vanishes outside $(-2 T, 2 T)(n \geqslant 1)$, and $\varphi_{n} * \widetilde{\varphi}_{n} \rightarrow \varphi * \widetilde{\varphi}$ uniformly on $(-\infty, \infty)$. Using Lebesgue's dominated convergence theorem, Theorem 1.1, and some standard facts about Fourier transforms we then get 


$$
\begin{aligned}
\overline{\langle\varphi * \widetilde{\varphi}}, \nu\rangle & =\lim _{n \rightarrow \infty}\left\langle\overline{\left\langle\varphi_{n} * \widetilde{\varphi}_{n}\right.}, \nu\right\rangle=\frac{1}{2 \pi} \lim _{n \rightarrow \infty}\left\langle\left|\hat{\varphi}_{n}\right|^{2}, \hat{\nu}\right\rangle \\
& =\frac{1}{2 \pi} \lim _{n \rightarrow \infty}\left\langle\left|\hat{\eta}_{n} \hat{\varphi}\right|^{2}, \hat{\nu}\right\rangle \geqslant \frac{1}{2 \pi}\left\langle|\hat{\varphi}|^{2}, \hat{\nu}\right\rangle,
\end{aligned}
$$

where the last inequality follows from Fatou's lemma. But this implies that

$$
\int_{-\infty}^{\infty}|\hat{\varphi}(\omega)|^{2} d \hat{v}(\omega)<\infty
$$

and so one can use Lebesgue's dominated convergence theorem to replace that inequality by

$$
\lim _{n \rightarrow \infty}\left\langle\left|\hat{\eta}_{n} \hat{\varphi}\right|^{2}, \hat{\nu}\right\rangle=\left\langle|\hat{\varphi}|^{2}, \hat{\nu}\right\rangle
$$

This completes the proof of Lemma 4.1.

By first stating and proving some more lemmas we can reduce the formal proof of Theorem 4.1 to a few lines.

Lemma 5.1. Let $\nu \in P D(-\infty, \infty), \varphi \in L^{\infty}(-\infty, \infty)$, and let (1) hold. Then

$$
\sup _{T>0} \int_{[0, T]} \bar{\psi}(t) \int_{[t-T, t]} \psi(t-s) d \nu(s) d t<\infty
$$

for every $\psi \in \Gamma(\varphi)$.

Proof of Lemma 5.1. Define $\varphi_{T}=\chi_{[0, T]} \varphi(T>0), K=$ $\sup _{T>0}\left\langle\overline{\left\langle\varphi_{T} * \widetilde{\varphi}_{T}\right.}, \nu\right\rangle$. Then by (1) and Lemma 4.1,

$$
\sup _{T>0}\left\langle\left|\hat{\varphi}_{T}\right|^{2}, \hat{\nu}\right\rangle=2 \pi K<\infty .
$$

Define $\varphi_{T, t}=\chi_{[0, T]}\left(\tau_{t} \varphi\right)(T, t>0)$. Then $\varphi_{T, t}=\varphi_{T+t}-\varphi_{t}(T, t>0)$, and thus by Minkowski's inequality,

$$
\sup _{T, t>0}\left\langle\left|\hat{\varphi}_{T, t}\right|^{2}, \hat{v}\right\rangle \leqslant 8 \pi K \text {. }
$$

Now fix $T>0$. Define the operator $B: L^{2}(0, T) \rightarrow(-\infty, \infty)$ by

$$
B(\eta)=[\overline{\langle\eta * \widetilde{\eta}}, \nu\rangle]^{1 / 2} \quad\left(\eta \in L^{2}(0, T)\right) .
$$

Then $B$ is (strongly) continuous, because $\nu$ restricted to $[-T, T]$ is finite. By Lemma 4.1 we also have $B(\eta)=\left[(1 / 2 \pi)\left\langle|\hat{\eta}|^{2}, \hat{v}\right\rangle\right]^{1 / 2}$, and using this representation and Minkowski's inequality one finds that $B$ is convex. This means that the set $A=\left\{\eta \in L^{2}(0, T) \mid B(\eta) \leqslant 2 \sqrt{K}\right\}$ is closed and convex, and hence also weakly closed in $L^{2}(0, T)$ [19, Theorem 3.12].

After these preliminaries we are ready to pick a function $\psi \in \Gamma(\varphi)$, and a sequence $t_{k} \rightarrow \infty$ such that $\tau_{t_{k}} \varphi \rightarrow \psi$ weak* in $L^{\infty}(-\infty, \infty)$. Define $\psi_{T}=$ $\chi_{[0, T]} \psi$. Then $\varphi_{T, t_{k}} \rightarrow \psi_{T}$ weakly in $L^{2}(0, T)$. By (5.1), $\varphi_{T, t_{k}} \in A(k \geqslant 1)$, and as $A$ is weakly closed we get $\psi_{T} \in A$, i.e. 


$$
\int_{[0, T]} \bar{\psi}(t) \int_{[t-T, t]} \psi(t-s) d v(s) d t \leqslant 4 K .
$$

Since this bound is independent of $T$, the proof of Lemma 5.1 is complete.

LEMMA 5.2. Let $\psi \in L^{\infty}(-\infty, \infty)$, and suppose that $\omega_{0}$ is an isolated point of $\sigma(\psi)$. Then $\psi$ can be written as $\psi(t)=\alpha e^{i \omega_{0} t}+\psi_{1}(t)(-\infty<t<\infty)$, where $\alpha$ is a nonzero constant, and $\psi_{1}$ satisfies $\omega_{0} \notin \sigma\left(\psi_{1}\right)$.

Proof of Lemma 5.2. Pick an open interval $I$ such that $I \cap \sigma(\psi)=\left\{\omega_{0}\right\}$, and then choose $\eta \in S$ such that $\sigma(\eta) \subset I$, and $\hat{\eta} \equiv 1$ in a neighborhood of $\omega_{0}$. Define $\varphi=\eta * \psi, \psi_{1}=\psi-\varphi$. Then $\sigma(\varphi)=\left\{\omega_{0}\right\}$, and hence $\varphi$ can be written as $\varphi(t)=\alpha e^{i \omega_{0} t}(-\infty<t<\infty)$, where $\alpha$ is a nonzero constant (first use [4, Theorem 4.11, p. 152], and then apply the inverse Fourier transform). That $\omega_{0} \notin \sigma\left(\psi_{1}\right)$ follows from the fact that $\hat{\varphi}=\hat{\eta} \hat{\psi}$ is equal to $\hat{\psi}$ in a neighborhood of $\omega_{0}$, i.e. $\hat{\psi}_{1}$ vanishes in a neighborhood of $\omega_{0}$. This completes the proof of Lemma 5.2.

Lemma 5.3. Let $\psi \in L^{\infty}(-\infty, \infty)$, and let $K \subset(-\infty, \infty)$ be a compact set satisfying $K \cap \sigma(\psi)=\varnothing$. Then

$$
\sup _{\omega \in K ; T>0}\left|\left(\chi_{[0, T]} \psi\right)^{\wedge}(\omega)\right|<\infty .
$$

Proof of Lemma 5.3. Pick $\eta \in S$ such that $\hat{\eta}(\omega)=1(\omega \in K)$, and $\sigma(\eta) \cap \sigma(\psi)=\varnothing\left(\right.$ cf. [19, Theorem 6.20]). Define $\psi_{T}=\left(X_{[0, T]} \psi\right) * \eta$. By $[19$, Theorems $7.2(\mathrm{c})$ and 7.5$]$ it clearly suffices to show that

$$
\sup _{T>0} \int_{-\infty}^{\infty}\left|\psi_{T}(t)\right| d t<\infty
$$

Using the definition of $\psi_{T}$ one immediately gets

$$
\left|\psi_{T}(t)\right| \leqslant \begin{cases}\|\psi\|_{\infty} \int_{-\infty}^{t}|\eta(s)| d s, & t \leqslant 0, \\ \|\psi\|_{\infty} \int_{t-T}^{\infty}|\eta(s)| d s, & t \geqslant T,\end{cases}
$$

where $\|\psi\|_{\infty}$ is the norm of $\psi$ in $L^{\infty}(-\infty, \infty)$. Thus

$$
\sup _{T>0}\left(\int_{-\infty}^{0}+\int_{T}^{\infty}\right)\left|\psi_{T}(t)\right| d t<\infty .
$$

Hence it only remains to show that

$$
\sup _{T>0} \int_{0}^{T}\left|\psi_{T}(t)\right| d t<\infty
$$

Since $\sigma(\eta) \cap \sigma(\psi)=\varnothing$, one has

$$
\int_{-\infty}^{\infty} \psi(t-s) \eta(s) d s=0 \quad(-\infty<t<\infty)
$$

(combine [19, Theorem 7.19(c)] with the Fourier inversion theorem), and thus 
one can also write $\psi_{T}$ as

This gives

$$
\psi_{T}(t)=-\left(\int_{-\infty}^{t-T}+\int_{t}^{\infty}\right) \psi(t-s) \eta(s) d s \quad(-\infty<t<\infty)
$$

and so

$$
\left|\psi_{T}(t)\right| \leqslant\|\psi\|_{\infty}\left(\int_{-\infty}^{t-T}+\int_{t}^{\infty}\right) \ln (s) \mid d s \quad(0 \leqslant t \leqslant T)
$$

$$
\sup _{T>0} \int_{0}^{T}\left|\psi_{T}(t)\right| d t \leqslant\|\psi\|_{\infty}\left(\int_{0}^{\infty} \int_{t}^{\infty}+\int_{-\infty}^{0} \int_{-\infty}^{t}\right)|\eta(s)| d s d t<\infty .
$$

This verifies (5.2), and completes the proof of Lemma 5.2.

Now we are finally ready for the proof of Theorem 4.1.

Proof of Theorem 4.1. We prove Theorem 4.1 indirectly by supposing that $\omega_{0}$ is an isolated point of $\sigma(\Gamma(\varphi))$, and showing that this implies (4.3), hence violates (4.1). Pick $\psi \in \Gamma(\varphi)$ such that $\omega_{0}$ is an isolated point of $\sigma(\psi)$. By Lemma 5.2 one then has $\psi(t)=\alpha e^{i \omega_{0} t}+\psi_{1}(t)(-\infty<t<\infty)$, where $\alpha \neq$ 0 , and $\omega_{0} \notin \sigma\left(\psi_{1}\right)$. Pick $\epsilon>0$ such that $\left[\omega_{0}-\epsilon, \omega_{0}+\epsilon\right] \cap \sigma\left(\psi_{1}\right)=\varnothing$. By (1) and Lemmas 4.1 and 5.1,

$$
\sup _{T>0} \int_{\left[\omega_{0}-\epsilon, \omega_{0}+\epsilon\right]}\left|\left(\chi_{[0, T]} \psi\right)^{-}(\omega)\right|^{2} d \hat{\nu}(\omega)<\infty
$$

or equivalently

$$
\sup _{T>0} \int_{\left[\omega_{0}-\epsilon, \omega_{0}+\epsilon\right]} \mid\left(\chi_{[0, T]} \psi_{1} \hat{)}(\omega)+\left.\alpha \frac{1-e^{-i T\left(\omega-\omega_{0}\right)}}{i\left(\omega-\omega_{0}\right)}\right|^{2} d \hat{\nu}(\omega)<\infty\right.
$$

(the second term inside the absolute value is defined by continuity at the point $\left.\omega_{0}\right)$. It follows from Lemma 5.3 that

$$
\sup _{T>0} \int_{\left[\omega_{0}-\epsilon, \omega_{0}+\epsilon\right]} \mid\left(\chi_{[0, T]} \psi_{1}\right)^{\wedge}(\omega)^{2} d \hat{\nu}(\omega)<\infty,
$$

and this combined with (5.3) and Minkowski's inequality gives

$$
\sup _{T>0} \int_{\left[\omega_{0}-\epsilon, \omega_{0}+\epsilon\right]} K_{T}\left(\omega-\omega_{0}\right) d \hat{\nu}(\omega)<\infty,
$$

with $K_{T}$ defined by (4.2). However, by Remark 4.1 this is equivalent to (4.3), and so the proof of Theorem 4.1 is complete.

6. On the condition (4.1). In the proof of Theorem 4.1 the condition (4.1) comes up very naturally (cf. Remark 4.2). However, as a condition on $\hat{v}$ the following equivalent form is more transparent:

Lemma 6.1. Let $\nu \in P D(-\infty, \infty)$. Then the condition (4.1) is equivalent to the following: either

$$
\hat{v}\left(\left\{\omega_{0}\right\}\right) \neq 0,
$$


or

$$
\int_{\omega \neq \omega_{0}}\left(\omega-\omega_{0}\right)^{-2} d \hat{\nu}(\omega)=\infty .
$$

Proof of Lemma 6.1. If both (6.1) and (6.2) are false, then (4.3) follows trivially. It is just as easy to see from (4.2) that (6.1) implies (4.1). The only nontrivial part of the proof is to show that (6.2) implies (4.1). So suppose that (6.2) holds. Take any (large) number $N$. Then by Remark 4.1 one can pick $\delta$, $0<\delta<1$, such that

$$
\left(\int_{\left[\omega_{0}-1, \omega_{0}-\delta\right]}+\int_{\left[\omega_{0}+\delta, \omega_{0}+1\right]}\right)\left(\omega-\omega_{0}\right)^{-2} d \hat{v}(\omega) \geqslant N .
$$

Suppose for the moment that

$$
\inf _{T>0}\left(\int_{\left[\omega_{0}-1, \omega_{0}-\delta\right]}+\int_{\left[\omega_{0}+\delta, \omega_{0}+1\right]}\right) \frac{\cos \left(T\left(\omega-\omega_{0}\right)\right)}{\left(\omega-\omega_{0}\right)^{2}} d \hat{v}(\omega) \leqslant 0
$$

Then, using also (4.2),

$$
\sup _{T>0}\left(\int_{\left[\omega_{0}-1, \omega_{0}-\delta\right]}+\int_{\left[\omega_{0}+\delta, \omega_{0}+1\right]}\right) K_{T}\left(\omega-\omega_{0}\right) d \hat{\nu}(\omega) \geqslant N,
$$

and so

$$
\sup _{T>0} \int_{-\infty}^{\infty} K_{T}\left(\omega-\omega_{0}\right) d \hat{\nu}(\omega) \geqslant N
$$

which implies (4.1), since $N$ was arbitrary. It thus only remains to prove (6.3).

The proof of (6.3) is based on the following idea (although we make no formal use of it): The point zero always belongs to the spectrum of a nonnegative function ( $\hat{\lambda}$ below), unless it vanishes identically. To be able to utilize this idea we define a new measure $\lambda$ by first defining a measure $\mu$ by

$$
d \mu(\omega)=\left\{\begin{array}{l}
\omega^{-2} d \hat{\nu}\left(\omega+\omega_{0}\right), \quad \delta \leqslant|\omega| \leqslant 1 \\
0, \quad|\omega|<\delta \text { or }|\omega|>1
\end{array}\right.
$$

and then setting $\lambda=\mu+\tilde{\mu}$. With these notations (6.3) becomes equivalent to

$$
\inf _{-\infty<T<\infty} \hat{\lambda}(T) \leqslant 0 .
$$

Suppose to get a contradiction that

$$
\inf _{-\infty<T<\infty} \hat{\lambda}(T)=\eta>0
$$

(or by slightly improving the argument below it would actually suffice to assume that $\eta \geqslant 0)$. Pick some $\varphi \in S$ such that $\varphi \geqslant 0, \hat{\varphi}(0)=1$, and $\sigma(\varphi) \subset(-\delta, \delta)$ (one can get such a function by rescaling the function $\varphi$ used in the proof of [22, Proposition 1.1]). Since the support of $\lambda$ does not intersect $(-\delta, \delta)$, we have $\langle\hat{\varphi}, \lambda\rangle=0$. But on the other hand, 


$$
\left.\langle\hat{\varphi}, \lambda\rangle=\langle\varphi, \hat{\lambda}\rangle=\int_{-\infty}^{\infty} \varphi(t) \hat{\lambda}(t) d t \geqslant \eta \int_{-\infty}^{\infty} \varphi(t) d t=\eta\right\rangle 0,
$$

and this contradiction completes the proof of Lemma 6.1.

It is now quite easy to show that (4.1) is equivalent to strict positivity of $\hat{v}$ at the point $\omega_{0}$, if $\hat{v}$ is smooth enough:

Proposition 6.1. Let $\nu \in P D(-\infty, \infty)$, and suppose that the (distribution) derivative of $\hat{\nu}$ is a Hölder continuous function (with some exponent $\alpha \in(0,1])$ in a neighborhood of a point $\omega_{0} \in(-\infty, \infty)$. Then (4.1) is equivalent to strict positivity of $\hat{\nu}$ at $\omega_{0}$.

Remark 6.1. Although the wording of Proposition 6.1 should be clear, some confusion may arise because of the fact that we have previously usually identified the distribution $\hat{v}$ with a measure, whereas in Proposition 6.1 it becomes natural to identify $\hat{\nu}$ (in some neighborhood of $\omega_{0}$ ) with a continuously differentiable function, whose derivative is Hölder continuous. If one prefers to stick to the interpretation of $\hat{v}$ as a measure, then Proposition 6.1 requires this measure to be absolutely continuous, and its derivative with respect to Lebesgue measure (i.e. the density function) to be continuously differentiable, and have a Hölder continuous derivative in a neighborhood of $\omega_{0}$.

Proof of Proposition 6.1. Pick $\epsilon$ sufficiently small, and identify $\hat{v}$ with a continuous function $u$ in $\left(\omega_{0}-\epsilon, \omega_{0}+\epsilon\right)$ (or define $u$ as the density function of the measure $\hat{\nu}$, cf. Remark 6.1). Since (6.1) is false it follows from Lemma 6.1 that (4.1) is equivalent to (6.2), and by Remark 4.1 we have (6.2) equivalent to

$$
\int_{\left(\omega_{0}-\epsilon\right)}^{\left(\omega_{0}+\epsilon\right)}\left(\omega-\omega_{0}\right)^{-2} u(\omega) d \omega=\infty
$$

The continuity of $u$ implies that $\hat{v}$ is strictly positive at $\omega_{0}$ if and only if $u\left(\omega_{0}\right)$ $>0$. If $u\left(\omega_{0}\right)>0$, then (6.4) trivially holds, so it only remains to show that (6.4) is false whenever $u\left(\omega_{0}\right)=0$. Let $u\left(\omega_{0}\right)=0$. The differentiability of $u$ at $\omega_{0}$ together with $u(\omega) \geqslant 0\left(\left|\omega-\omega_{0}\right|<\epsilon\right)$ then forces $u^{\prime}\left(\omega_{0}\right)=0$ (prime denotes differentiation). This together with the Hölder continuity of $u^{\prime}$ then implies that the function $u(\omega)\left(\omega-\omega_{0}\right)^{-2}$ is integrable in $\left(\omega_{0}-\epsilon, \omega_{0}+\epsilon\right)$, i.e. (6.4) is false, and the proof of Proposition 6.1 is complete.

Our next proposition gives a slightly different criterion on $\hat{v}$ to be "smooth enough" for (4.1) to be equivalent to strict positivity of $\hat{\nu}$ at $\omega_{0}$. Note that in this case the (distribution) derivative of $\hat{\nu}$ is not necessarily Hölder continuous (although it is continuous).

Proposition 6.2. Let $\nu \in P D(-\infty, \infty)$, and suppose that

$$
\int_{0}^{\infty}|\nu|((t, \infty)) d t<\infty \text {. }
$$


Then (4.1) is equivalent to strict positivity of $\hat{v}$ at $\omega_{0}$ for any $\omega_{0} \in(-\infty, \infty)$.

Note that (6.5) implies that the measure $\nu$, restricted to $[0, \infty)$, is finite, and has a finite moment.

Proof of Proposition 6.2. The condition (6.5) together with [22, Theorem 1.1] implies that

$$
\int_{-\infty}^{0}|\nu|((-\infty, t)) d t<\infty
$$

In particular, $|\nu|((-\infty, \infty))<\infty$, so one can identify $\hat{v}$ with a continuous function $u$. Thus $\hat{\nu}$ is strictly positive at a point $\omega_{0}$ if and only if $u\left(\omega_{0}\right)>0$. As before, $u\left(\omega_{0}\right)>0$ implies (6.2), which in turn implies (4.1). Thus we only have to show that $u\left(\omega_{0}\right)=0$ implies (4.3). By Remark 4.2, the condition (4.3) is equivalent to

$$
\sup _{T>0} \int_{[0, T]} \int_{[t-T, t]} e^{-i \omega_{0} s} d \nu(s) d t<\infty
$$

Since $u\left(\omega_{0}\right)=0$, i.e. $\int_{-\infty}^{\infty} e^{-i \omega_{0} s} d v(s)=0$, this can be written as

But

$$
\sup _{T>0}\left|\int_{0}^{T}\left(\int_{(-\infty, t-T)}+\int_{(t, \infty)}\right) e^{-i \omega_{0} s} d \nu(s) d t\right|<\infty .
$$

$$
\begin{aligned}
& \left|\int_{0}^{T}\left(\int_{(-\infty, t-T)}+\int_{(t, \infty)}\right) e^{-i \omega_{0} s} d \nu(s) d t\right| \\
& \leqslant \int_{-\infty}^{T}|\nu|((-\infty, t-T)) d t+\int_{0}^{\infty}|\nu|((t, \infty)) d t \\
& \quad=\int_{-\infty}^{0}|\nu|((-\infty, t)) d t+\int_{0}^{\infty}|\nu|((t, \infty)) d t<\infty,
\end{aligned}
$$

where the finiteness follows from (6.5)-(6.6). This completes the proof of Proposition 6.2 .

Combining Theorems 3.1 and 4.2 with Propositions 6.1 and 6.2 one now gets:

COROllaRY 6.1. Let $\nu \in P D(-\infty, \infty)$, and suppose that either the (distribution) derivative of $\hat{v}$ is locally Hölder continuous on $(-\infty, \infty)$, or that $(6.5)$ is true. Then every $\varphi \in L^{\infty}(-\infty, \infty)$ for which (1) holds satisfies $\tau_{t} \varphi \rightarrow 0$ weak ${ }^{*}$ in $L^{\infty}(-\infty, \infty)$ as $t \rightarrow \infty$ if and only if $\nu \in \operatorname{SPD}(-\infty, \infty)$.

EXAMPLE 6.1. Let $\nu \in P D(-\infty, \infty)$, and suppose that $d \hat{\nu}(\omega)=$ $\left|\omega-\omega_{1}\right|^{\alpha} c(\omega) d \omega(-\infty<\omega<\infty)$, where $c$ is continuous and strictly positive on $(-\infty, \infty)$, and $\alpha>-1$. It follows from Definitions 1.3-1.4 that $\nu \in \operatorname{SPD}(-\infty, \infty)$ (i.e. $Z(\nu)=\varnothing$ ) if and only if $\alpha \leqslant 0$. Thus for these values of $\alpha$ one can conclude from Theorem 3.1 and Lemma 2.1 that whenever a function $\varphi \in L^{\infty}(-\infty, \infty)$ satisfies (1) then $\tau_{t} \varphi \rightarrow 0$ weak $^{*}$ in $L^{\infty}(-\infty, \infty)$ as $t \rightarrow \infty$. However, the same 
statement is in fact true for a much larger set of values of $\alpha$, i.e. for $\alpha \leqslant 1$. This follows from Corollary 4.1 , because (6.2), or equivalently (4.1), then holds with $\omega_{0}=\omega_{1}$ even though $Z(\nu)$ is nonempty $\left(Z(\nu)=\left\{\omega_{1}\right\}\right)$ as soon as $\alpha>0$. On the other hand, when $\alpha>1$ then it is no longer true that every $\varphi$ for which (1) holds must satisfy $\tau_{t} \varphi \rightarrow 0$ weak* in $L^{\infty}(-\infty, \infty)$ as $t \rightarrow \infty$, because in this casse (4.1) is violated for $\omega_{0}=\omega_{1}$, and one can apply the "only if" part of Corollary 4.1.

EXAMPLE 6.2. Here we shall show that (4.1) can be satisfied at a point $\omega_{1}$ even in some (extreme) cases where the density function $u$ of the measure $\hat{v}$ has a zero at $\omega_{1}$, and also is continuously differentiable (in the previous example the derivative of $u(\omega)=\left|\omega-\omega_{1}\right|^{\alpha} c(\omega)$ does not exist at $\omega_{1}$ whenever (4.1) holds for $\omega_{0}=\omega_{1}$, i.e. whenever $\left.\alpha \leqslant 1\right)$. This time take

$d \hat{\nu}(\omega)=u(\omega) d \omega, \quad u(\omega)=\left|\omega-\omega_{1}\right|\left(1+\left(\log \left|\omega-\omega_{1}\right|\right)^{2}\right)^{-1 / 2} c(\omega)(-\infty<\omega<\infty)$,

where $c$ is some strictly positive and continuously differentiable function on $(-\infty, \infty)$ (also suppose that $c$ is sufficiently small at infinity so that $u$ is the Fourier transform of a measure). Then

$$
\int_{\omega_{1}-1 / 2}^{\omega_{1}+1 / 2}\left(\omega-\omega_{1}\right)^{-2} u(\omega) d \omega=\infty
$$

and thus (6.2) holds, which implies (4.1). It is not difficult to see that $u\left(\omega_{1}\right)=$ 0 , and that $u$ is continuously differentiable on $(-\infty, \infty)$. The derivative is, of course, not Hölder continuous (cf. Proposition 6.1). Also the measure $\nu$ cannot possibly satisfy (6.5) (see Proposition 6.2).

7. Applications to a Volterra equation. The results in $\S \S 3,4$ and 6 can be used to study the asymptotic behavior of the bounded solutions of the nonlinear Volterra integrodifferential equation

$$
x^{\prime}(t)+\int_{[0, t]} g(x(t-s)) d \mu(s)=f(t) \quad(0 \leqslant t<\infty) ; x(0)=x_{0} .
$$

Here $g$ and $f$ are real-valued functions, $\mu$ is a real, locally finite measure, $x_{0}$ is a real constant, and $x$ is the unknown solution, which is required to be locally absolutely continuous and satisfy (7.1) a.e. All our applications are based on the following lemma:

LEMMA 7.1. Let $\mu$ be a locally finite measure with support in $[0, \infty)$, let $g \in C(-\infty, \infty), f \in L^{1}(0, \infty)$, and let $x$ be a bounded solution of $(7.1)$ on $[0, \infty)$. Define $\varphi(t)=g(x(t))(t \geqslant 0)$. Then (1) is satisfied with $\nu=\mu+\tilde{\mu}$.

Although we have not seen Lemma 7.1 stated explicitly before, different versions of it have been used e.g. in [2], [11], [12], [14]-[16], [21] and [22]. The proof is not complicated: Multiply $(7.1)$ by $g(x(t))$, integrate over $[0, T]$ (cf. [21, §2]), and then use [22, Lemma 1.1]. 
We now get

Proposition 7.1. Let the assumption of Lemma 7.1 hold, and define $\varphi$ and $\nu$ as in Lemma 7.1. Then the conclusions of Theorems 3.1, 4.1, 4.3 and Corollaries 3.1, 3.2, 3.3 are true, provided $\nu$ satisfies the particular conditions on $\nu$ (and $Z(\nu)$ ) required in those theorems and corollaries (if $Z(\nu)$ is not compact in Corollary 3.1, then one must also suppose that $\varphi$ is uniformly continuous).

This is a consequence of Lemma 7.1, and it summarizes the results of \$§34 as they immediately apply to equation (7.1).

We shall illustrate the force of Proposition 7.1 by continuing Example 6.1. Define

$$
a(t)=e^{-|t|}-\frac{1}{\pi} \int_{-\infty}^{\infty} e^{-|t-s|} s^{-2}(1-\cos (s)) d s \quad(-\infty<t<\infty) .
$$

Then $a \in L^{1}(-\infty, \infty)$, and

$$
\int_{-\infty}^{\infty} \cos (\omega t) a(t) d t= \begin{cases}2|\omega| /\left(1+\omega^{2}\right), & |\omega| \leqslant 1, \\ 2 /\left(1+\omega^{2}\right), & |\omega|>1 .\end{cases}
$$

Thus in particular, defining $d \nu(t)=a(t) d t(-\infty<t<\infty)$ one has $Z(\nu)=\{0\}$, and (6.2) holds with $\omega_{0}=0$. Consider the equation

$$
x^{\prime}(t)+\int_{0}^{t} g(x(t-s)) a(s) d s=f(t) \quad(0 \leqslant t<\infty) ; x(0)=x_{0} .
$$

Here let $f \in L^{1}(0, \infty), g \in C(-\infty, \infty)$, and let $x$ be a bounded solution of (7.2) on $[0, \infty)$. By $[22$, Theorem 6.3$], x$ is uniformly continuous on $[0, \infty)$, and thus also the function $\varphi(t)=g(x(t))(t \geqslant 0)$ is uniformly continuous on $[0, \infty)$. It then follows from Proposition 7.1 (the part referring to Theorem 4.3) and Lemmas $2.1-2.2$ that $g(x(t)) \rightarrow 0$ as $t \rightarrow \infty$. The assumption $f \in L^{1}(0, \infty)$ is very sharp, i.e. it cannot be replaced by $f(t)=O\left(t^{-1}\right)$ as $t \rightarrow \infty$ (provided $g \neq$ 0 ), as the following specific choice of $x$ and $f$ shows: Pick $x_{0}$ such that $g\left(x_{0}\right)$ $\neq 0$, and define $x(t)=x_{0}, f(t)=g\left(x_{0}\right) \int_{0}^{t} a(s) d s(t \geqslant 0)$. Then (7.2) holds, and, of course, $g(x(t)) \nrightarrow 0$ as $t \rightarrow \infty$. Since $\int_{0}^{\infty} a(s) d s=0$, we also have $f(t)$ $=-g\left(x_{0}\right) \int_{t}^{\infty} a(s) d s$. But

$$
\begin{aligned}
|a(t)| & \leqslant e^{-t}+\frac{1}{\pi}\left(\int_{-\infty}^{t / 2}+\int_{t / 2}^{\infty}\right) e^{-|t-s|} s^{-2}(1-\cos (s)) d s \\
& \leqslant e^{-t}+e^{-t / 2}+\frac{16}{\pi} t^{-2}=O\left(t^{-2}\right) \quad(t \rightarrow \infty),
\end{aligned}
$$

which then gives $f(t)=O\left(t^{-1}\right)(t \rightarrow \infty)$, as we claimed above.

Finally, we also note that even in the linear case $g(\xi)=\xi(-\infty<\xi<\infty)$ the preceding result is new. The resolvent equation 


$$
r^{\prime}(t)+\int_{0}^{t} r(t-s) a(s) d s=0 \quad(0 \leqslant t<\infty) ; r(0)=1
$$

(with the same kernel $a$ as before) is a special case of (7.2), and thus one finds that $r(t) \rightarrow 0$ as $t \rightarrow \infty$ (first apply e.g. [22, Theorem 6.1] to show that $r$ is bounded on $[0, \infty))$. Recently much work has been devoted to the problem whether or not the resolvent $r$ of a kernel $a$ is integrable (see e.g. [1] and [20]), and e.g. when $a \in L^{1}(0, \infty)$, as is the case above, this problem has been completely solved [1, Theorem 3.5] (some corresponding results for the nonlinear equation are also known; see [6, Theorem 2.5] and [13, Theorem 3]). For this particular kernel the critical Laplace transform condition [1, Definition 2.3] is violated at the origin, and hence $r \notin L^{1}(0, \infty)$ (this is the easy part of [1, Theorem 3.5]). However, much more is true. In fact $r \notin L^{p}(0, \infty)$ for any $p<\infty$, i.e. the convergence to zero is extremely slow. This follows from the counterexample which we constructed above on a function $f$ satisfying $f(t)=O\left(t^{-1}\right)$ as $t \rightarrow \infty$ (in particular, $f \in L^{q}(0, \infty)$ for every $q>1$ ), such that the solution $x$ of (7.2) (with $g(\xi)=\xi$ ) does not tend to zero as $t \rightarrow \infty$. This behavior would be impossible if $r \in L^{p}(0, \infty)$ for some $p<\infty$, as one can see from the variation of constants formula $[1$, line (2.2)] (take $q$ to be the conjugate exponent of $p$; then $(r * f)(t) \rightarrow 0$ as $t \rightarrow \infty)$.

Acknowledgments. The author wishes to thank Professor John Nohel for his encouragement and his suggested improvements in this presentation, and Professor Daniel Shea for his guidance and support in the early stages of this work. This paper is based on the last part of the author's Ph.D. dissertation submitted to the University of Wisconsin, Madison.

\section{REFERENCES}

1. S. I. Grossman and R. K. Miller, Nonlinear Volterra integrodifferential systems with $L^{1}$-kernels, J. Differential Equations 13 (1973), 551-566. MR 50 \#915.

2. A. Halanay, On the asymptotic behavior of the solutions of an integro-differential equation, J. Math. Anal. Appl. 10 (1965), 319-324. MR 31 \#579.

3. K. B. Hannsgen, On a nonlinear Volterra equation, Michigan Math. J. 16 (1969), 365-376. MR 40 \#3225.

4. Y. Katznelson, An introduction of harmonic analysis, Wiley, New York, 1968. MR $40 \# 1734$.

5. J. J. Levin, On a nonlinear Volterra equation, J. Math. Anal. Appl. 39 (1972), 458-476. MR 46 \#124.

6. On some geometric structures for integrodifferential equations, Advances in Math. (to appear).

7. J. J. Levin and J. A. Nohel, Note on a nonlinear Volterra equation, Proc. Amer. Math. Soc. 14 (1963), 924-929. MR 28 \#37.

MR 29 \#445.

8. - On a nonlinear delay equation, J. Math. Anal. Appl. 8 (1964), 31-44.

9. - Perturbations of a nonlinear Volterra equation, Michigan Math. J. 12 (1965), 431-447. MR 32 \#336.

10. J. J. Levin and D. F. Shea, On the asymptotic behavior of the bounded solutions 
of some integral equations. I-III, J. Math. Anal. Appl. 37 (1972), 42-82, 288-326, 537575. MR 46 \#5971.

11. S.-O. Londen, The qualitative behavior of the solutions of a nonlinear Volterra equation, Michigan Math. J. 18 (1971), 321-330. MR 45 \#2431.

12. - On a nonlinear Volterra integral equation, J. Differential Equations 14 (1973), 106-120. MR 49 \#5745.

13. - On the variation of the solutions of a nonlinear integral equation, $J$.

Math. Anal. Appl. 52 (1975), 430-449.

14. R. C. MacCamy, Nonlinear Volterra equations on a Hilbert space, J. Differential Equations 16 (1974), 373-393.

15. R. C. MacCamy and J. S. W. Wong, Stability theorems for some functional equations, Trans. Amer. Math. Soc. 164 (1972), 1-37. MR 45 \#2432.

16. J. A. Nohel and D. F. Shea, Frequency domain methods for Volterra equations, Advances in Math. (to appear).

17. H. L. Royden, Real analysis, 2nd ed., Macmillan, London, 1968.

18. W. Rudin, Real and complex analysis, 2nd ed., McGraw-Hill Ser. in Higher Math., McGraw-Hill, New York, 1974. MR 49 \#8783.

19. - Functional analysis, McGraw-Hill Ser. in Higher Math., McGraw-Hill, New York, 1973.

20. D. F. Shea and S. Wainger, Variants of the Wiener-Lévy theorem, with applications to stability problems for some Volterra integral equations, Amer. J. Math. 97 (1975), 312-343.

21. O. J. Staffans, Nonlinear Volterra integral equations with positive definite kernels, Proc. Amer. Math. Soc. 51 (1975), 103-108.

22. Positive definite measures with applications to a Volterra equation, Trans. Amer. Math. Soc. 218 (1976), 219-237.

INSTITUTE OF MATHEMATICS, HELSINKI UNIVERSITY OF TECHNOLOGY, SF-02150 ESPOO 15, FINLAND 